\title{
Molecular Dynamics in Formation Process of SWNTs
}

\author{
Shigeo Maruyama and Yasushi Shibuta \\ Department of Mechanical Engineering, The University of Tokyo \\ 7-3-1 Hongo, Bunkyo-ku, Tokyo 113-8656, JAPAN
}

\section{Abstract}

The formation mechanism of single-walled carbon nanotubes (SWNTs) was studied with molecular dynamics simulations. Theoretical contributions to the formation mechanism of carbon nanotubes are not straightforward since the system size and time scale are far beyond the simple simulation of whole process. Several previous classical molecular dynamics calculations have examined the specific points of formation mechanism usually with the Brenner potential [1] for carbon only system. However the roles of metal catalysis have not been discussed since a reliable classical potential function between metal and carbon has not been known. We had constructed the classical potential function between carbon and several metal atoms (La, Sc and Ni) based on DFT calculations of small metal-carbon binary clusters [2]. Using these potential functions, molecular dynamics simulation was performed with carbon and Ni system for the precursor clusters of SWNTs.

The initial stage of the growth process of metal-carbon binary clusters was simulated with the same technique as our previous simulation for endohedral metallofullerene [2]. As the initial condition, the completely random vapor mixture of 2500 carbon and $25 \mathrm{Ni}$ atoms were allocated in a $585 \AA$ cubic fully-periodic simulation cell. The high density of the system was compensated with the special temperature control method at $3000 \mathrm{~K}$ [2]. After $6 \mathrm{~ns}$ simulation, many relatively large clusters up to about 100 carbon atoms and a few metal atoms were observed. Carbon clusters tended to be spherical random cage structure with a few metal atoms at around the defect vacancy, which prevented the cage structure from the complete closure.

In the next stage, we slowly shrank the simulation cell to enhance the collisions with the shrinking rate set as the $6 \times 10^{-5} \AA$ per time step (about $12 \mathrm{~m} / \mathrm{s}$ ), which was much slower than the typical translational velocity of clusters. As shown in Figure 1, a large cluster with some tubular structure was obtained after the collision of clusters. To accelerate the effect of annealing, we applied higher temperature at $2000 \mathrm{~K}$ than the typical experimental condition [3]. Even though the structure shown in Figure 1 is rather ugly, one can see that the tubular structure has grown longer by the collision and the coalescence. Ni atoms were slowly 


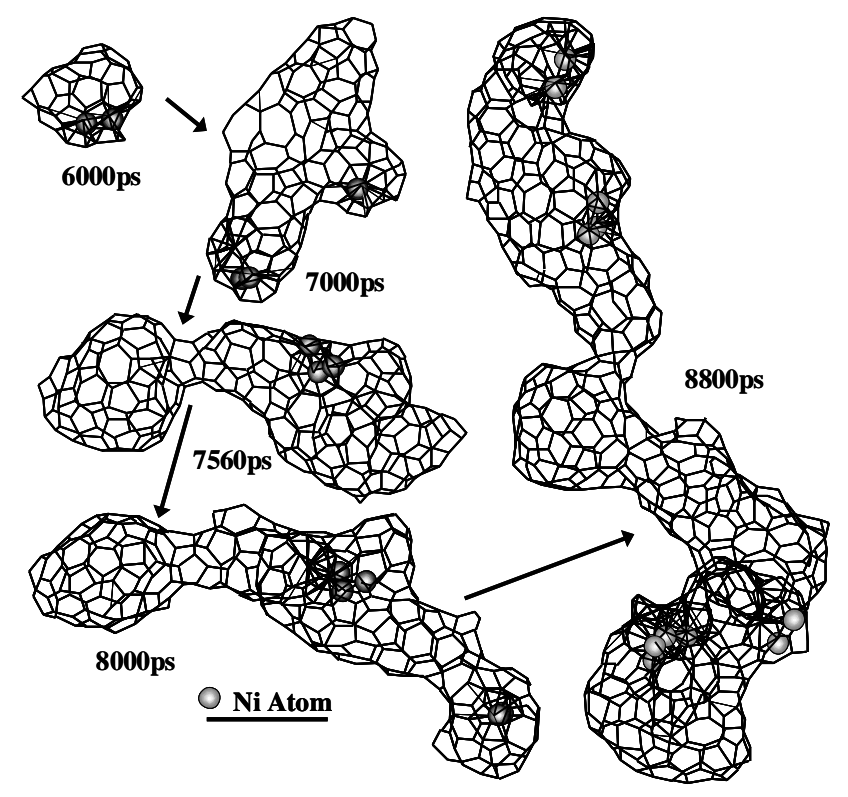

Fig. 1 The molecular dynamics simulation of the growth process of a large tubular cluster.

assembling to form Ni clusters, and they were diffusing around until finding the most stable position at the hemi-half-fullerene cap area. Since the narrow part of the tubular structure was usually Ni free, the tubular structure can be annealed to SWNT structure.

From the molecular dynamics simulation, the catalytic effect of $\mathrm{Ni}$ atoms on the formation of SWNT may be explained as follows. At the initial clustering stage, Ni atoms tend to stay at around the defect vacancy of carbon-caged structure, and prevent the cage from closing to fullerene. Then, the random cage carbon clusters can make further growth by colliding with each other at the large defect area of the cage. The collision leads to the elongated tubular structure as shown in Figure 1. Given the enough time for diffusion of metal atoms and network annealing, we expect that the structure becomes a straight SWNT with metal clusters at each end.

\section{References}

[1] D. W. Brenner, Phys. Rev. B, 42, 9458(1990).

[2] Y. Yamaguchi and S. Maruyama, Eur. Phys. J. D, 9, 385(1999).

[3] A. Thess, et al., Science, 273, 483 (1996).

Corresponding Author: Shigeo Maruyama

Department of Mechanical Engineering, The University of Tokyo, 7-3-1 Hongo, Bunkyo-ku, Tokyo 113-8656, Japan

Tel: +81-3-5841-6421, Fax: +81-3-5800-6983, E-mail: maruyama@ photon.t.u-tokyo.ac.jp 